\title{
Cross-Sectional Transmission Electron Microscopy Specimen Preparation Technique by Backside Ar Ion Milling
}

*Correspondence to:

Yoo $\mathrm{JH}$,

Tel: +82-42-366-1703

Fax: +82-42-366-1990

E-mail: jhyoo@nnfc.re.kr

Received December 4, 2015

Revised December 9, 2015

Accepted December 9, 2015

\author{
Jung Ho Yoo*, Jun-Mo Yang \\ Department of Measurement \& Analysis, National Nanofab Center, Daejeon 34141, Korea
}

\begin{abstract}
Backside Ar ion milling technique for the preparation of cross-sectional transmission electron microscopy (TEM) specimens, and backside-ion milling combined with focused ion beam (FIB) operation for electron holography were introduced in this paper. The backside Ar ion milling technique offers advantages in preparing cross-sectional specimens having thin, smooth and uniform surfaces with low surface damages. The back-side ion milling combined with the FIB technique could be used to observe the two-dimensional $p-n$ junction profiles in semiconductors with the sample quality sufficient for an electron holography study. These techniques have useful applications for accurate TEM analysis of the microstructure of materials or electronic devices such as arrayed hole patterns, threedimensional integrated circuits, and also relatively thick layers $(>1 \mu \mathrm{m})$.
\end{abstract}

Key Words: Backside Ar ion milling, Curtaining effect, Transmission electron microscopy specimen preparation, Electron holography, Focused ion beam

\section{INTRODUCTION}

Transmission electron microscopy (TEM) is one of the most useful techniques to provide analytical informations such as the morphology, microstructure, interfaces and chemical composition at the nanometer scale and has been widely used for the characterization of materials and devices. However, in order to obtain accurate and precise information using TEM studies, specimens have to be very thin for transferring the highly accelerated electrons and require clean and smooth surfaces in the analysis region.

Until now, several techniques have been widely used to prepare cross-sectional TEM specimens, such as ion milling (John \& Robert, 1984; Chew and Cullis, 1987), focused ion beam (FIB) (Stevie et al., 1995; Giannuzzi \& Stevie, 1999; Orloff et al., 2003) and mechanical wedge polishing (Mkhoyan et al., 2006; Okuno et al., 2008), and so on. FIB technique is effective for preparing specimens like electronic devices or patterned devices, since it is easy to prepare specimens at a specific site with a constant thickness using this technique.
However, because of the bombardment by Ga ions during FIB operation, surfaces of the specimen were inevitably damaged, including the formation of an amorphous phase, which decreased the detection limit and accuracy of the TEM analysis. And also, specimens prepared by FIB have a small field of view compared with those prepared by Ar ion milling. Mechanical wedge polishing was useful to obtain a smooth sample surface without any ion damage but this technique is difficult to prepare the specimen and extract a specific site.

Ion milling, which is the representative specimen preparation method, can be used to strip off the specimen surface by sputtering using inert gas ions (e.g., Ar, Xe) at an oblique angle. Ion milling is very effective for obtaining a wide and thin specimen and has been applied to both TEM crosssectional and plane-view observations, although it is difficult to selectively control the position at the micrometer scale. However, when TEM specimens are prepared utilizing the conventional Ar ion milling technique, a thickness variation known as the curtaining effect is generated. The curtaining effect prevents precise TEM observations; it is caused by

(a) This is an open-access article distributed under the terms of the Creative Commons Attribution Non-Commercial License (http://creativecommons.org/licenses/by-nc/4.0) which permits unrestricted noncommercial use, distribution, and reproduction in any medium, provided the original work is properly cited.

Copyrights @ 2015 by Korean Society of Microscopy 
the different milling rates of materials in, for example, the patterned transistor or the different crystal components. In this paper, we will introduce the backside Ar ion milling technique to reduce the curtaining effect for cross-sectional TEM specimens. In addition, we also introduce the backsideion milling method combined with FIB operation, which is a very promising specimen preparation technique for the reliable and quantitative analysis of dopant profiling with electron holography.

\section{METHODS AND RESULTS}

\section{Specimen Preparation by Backside-lon Milling Technique}

The process of backside-ion milling was similar to the conventional cross-sectional preparation technique (Abrahams \& Buiocchi, 1974; Traeholt et al., 1993) which consisted of wafer bonding, slicing, disc cutting, mechanical thinning, dimple grinding, and a final ion milling process as shown in Fig. 1A. Details of the process of specimen preparation are summarized as follows. First, pieces for crosssectional observation were bonded face-to-face using epoxy resin and sliced about $1 \mathrm{~mm}$ in order to enable facile cutting into a disc shape. Subsequently, the specimen was finely polished from both sides until the thickness reached 50 to 70 $\mu \mathrm{m}$ and carried out dimple grinding until the thickness of the central part reached about $5 \mu \mathrm{m}$. Finally the specimen was ion milled in such a way that Ar ions etched only from the substrate side of the specimen as shown in Fig. 1A.

Backside-ion milling was carried out using precision ion preparation system (Gatan, USA) and the prepared specimens were observed using a $200 \mathrm{kV}$ field emission-TEM (FE-TEM, JEM-2100F HR; JEOL, Japan).

Fig. $1 \mathrm{~B}$ and $\mathrm{C}$ show optical microscope images of a specimen after the back-side ion milling process. Since Ar ions came only from the substrate side, in the specimen region, it appears to be uniformly or gradually changing the thickness despite observing Newton fringes in which the thickness of a specimen is steeply changing (Preble et al., 2002) as indicated by the black arrow in Fig. 1B. Meanwhile at the dummy wafer above the specimen region, shown in Fig. 1C, there are thickness changes due to the curtaining effect as indicated by the white box.

Fig. 2 shows a sample with 40 pairs of silicon oxide/nitride multi-layers with the total thickness of the multilayer being 1.2 $\mu \mathrm{m}$. When the total thickness of the layer on the wafer is about several tens of nanometers, it is relatively easy to prepare the cross-sectional TEM specimen using the conventional ion milling technique. However, with increasing layer thickness of the specimen, it becomes difficult to obtain specimens with

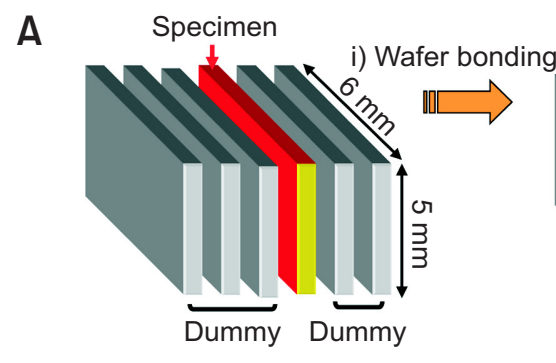

iv) Dimple grinding
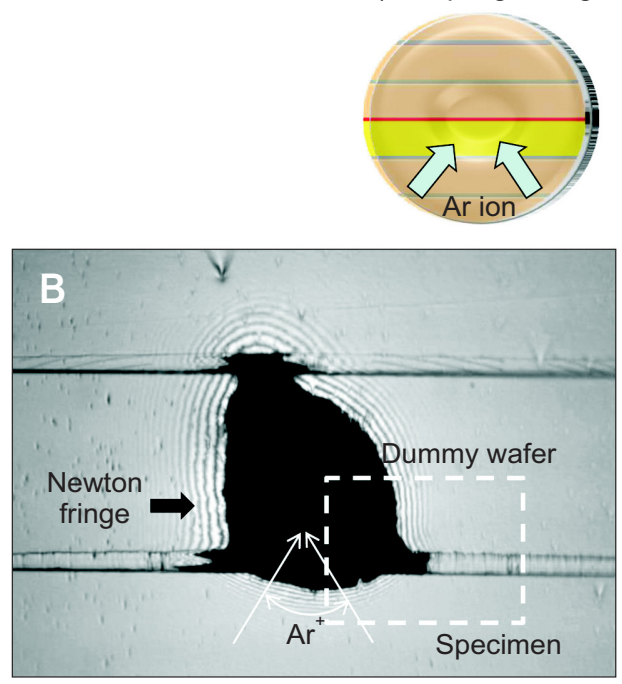

ii) Slicing

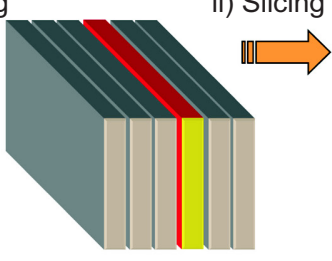

iii) Disc cutting \& thinning
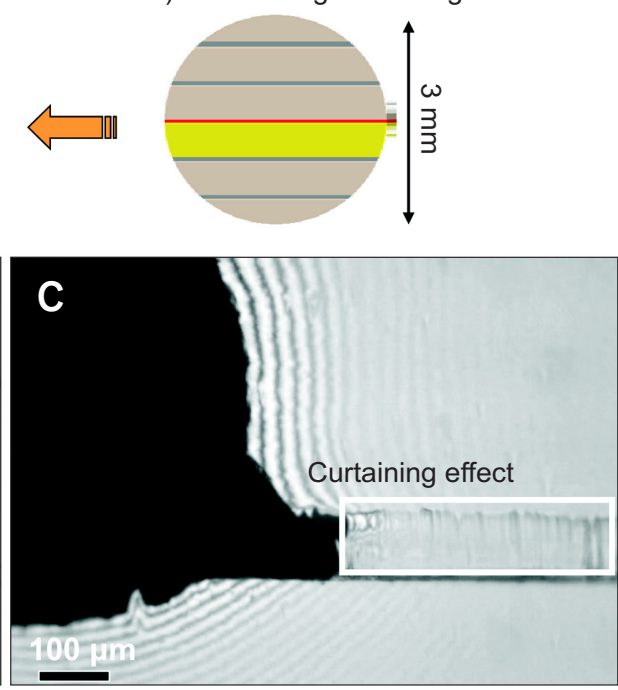

Fig. 1. (A) Schematic illustrations show cross-sectional transmission electron microscopy specimen preparation procedure for the backside-ion milling technique. Optical microscope images of the cross-sectioned specimen after backside-ion milling (B) and the enlarged image of the dotted box in Fig. 1B (C). 

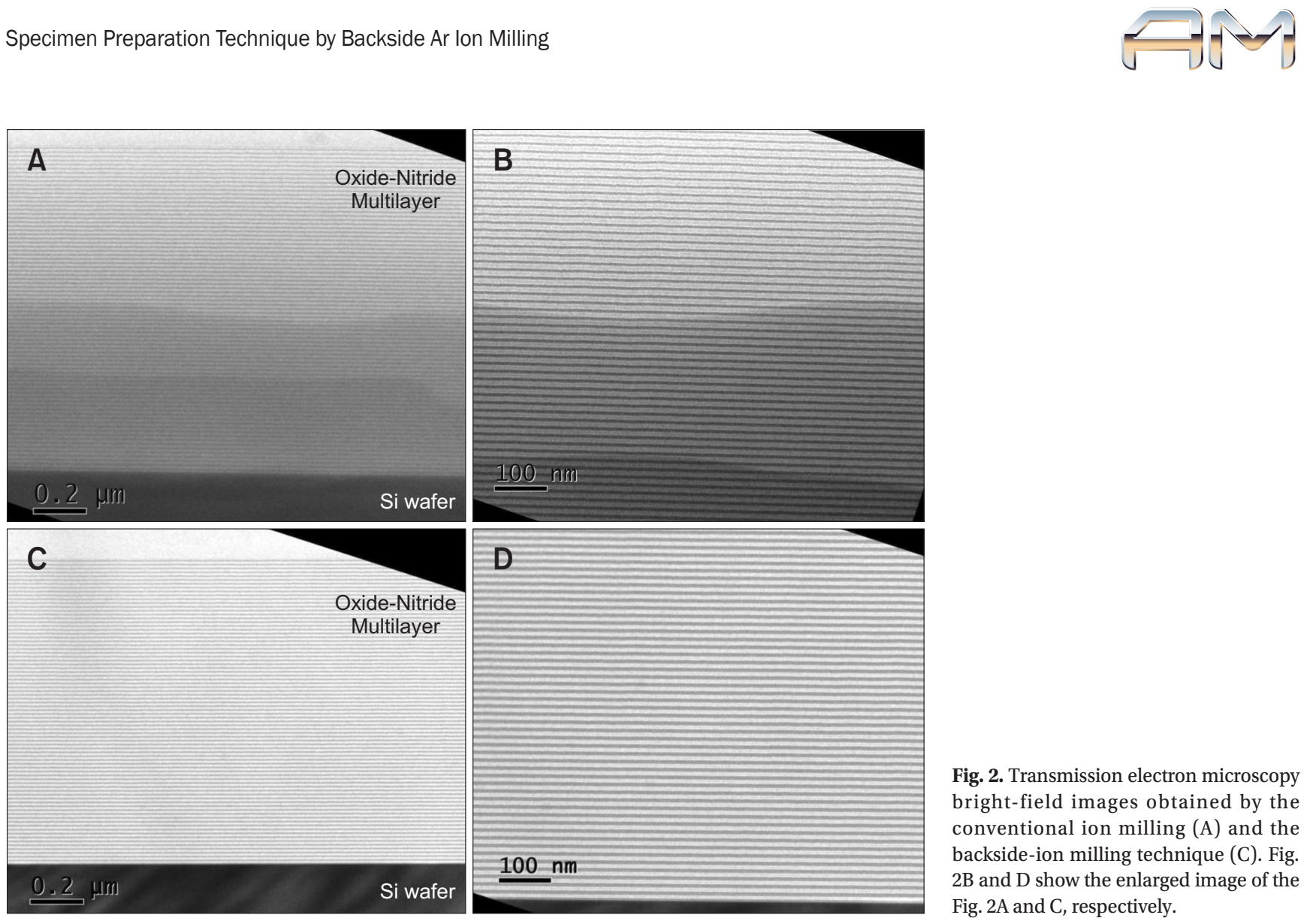

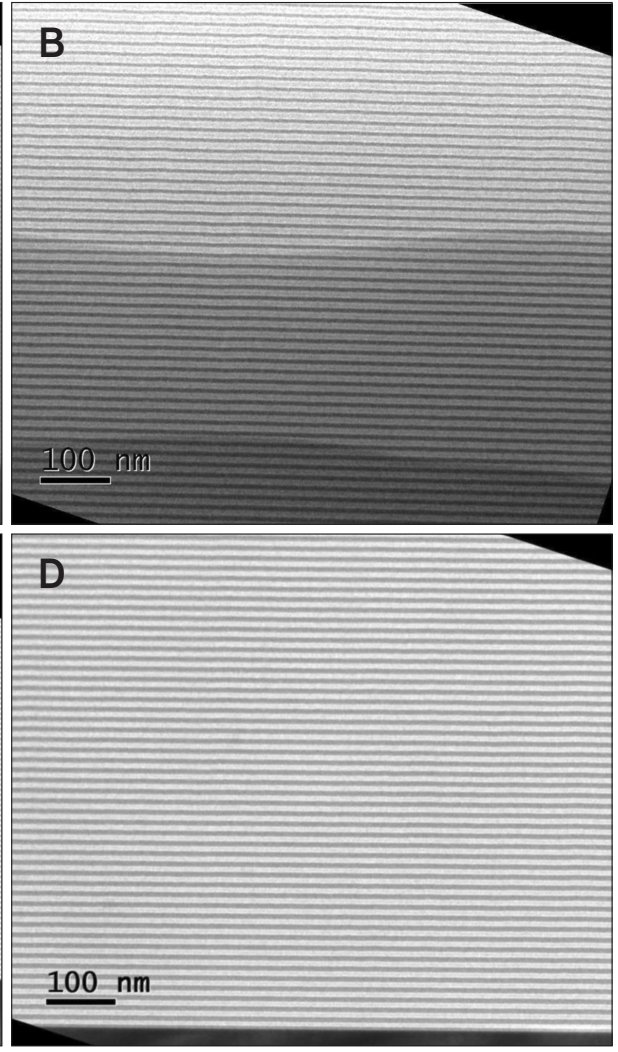

Fig. 2. Transmission electron microscopy bright-field images obtained by the conventional ion milling (A) and the backside-ion milling technique (C). Fig. $2 \mathrm{~B}$ and $\mathrm{D}$ show the enlarged image of the Fig. $2 \mathrm{~A}$ and $\mathrm{C}$, respectively.
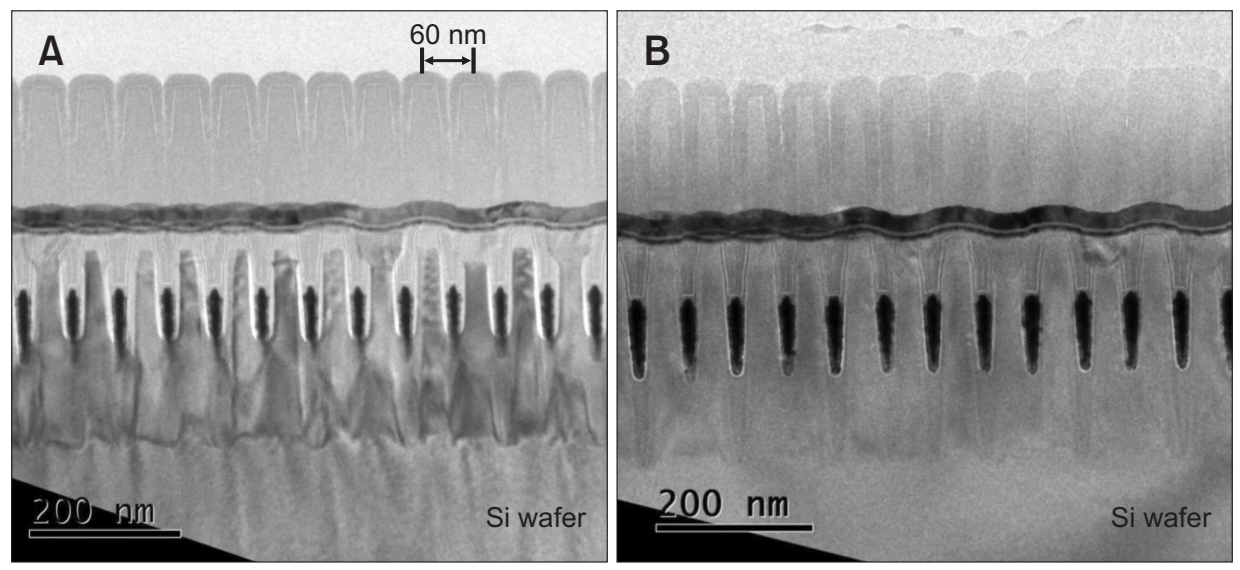

Fig. 3. Transmission electron microscopy bright-field images of the patterned specimen; prepared by the conventional ion milling (A) and the backside-ion milling (B) technique. uniform thickness because of the randomly scattered Ar ions. Fig. 2A and B show the TEM bright-field images prepared by conventional ion milling technique. Images show very large fluctuations in the thickness due to the irregularly scattered Ar ions. In contrast, when we used the backside-ion milling technique, there was little thickness change, and a thin and uniform surface could be observed as shown in Fig. 2C and D. As the process size in semiconductor devices (e.g., flash memory, process device or three-dimensional [3D] integrated circuits etc.) shrinks in nano-scale, it has been required to prepare extremely thin specimens without damaged layers.
Until now, to prepare the cross-sectional TEM specimen of the electronic devices, FIB is mainly used because it is able to fabricate a cross-sectional specimen at the specific site. However, when preparing the TEM specimen for some kinds of devices, including patterns with a high aspect ratio or with nano-sized hole arrays, the FIB technique is hard to be applied because the TEM specimen required for observing these patterns need to be a very thin foil.

In these cases, backside-ion milling becomes an appropriate technique for making such a thin foil. Fig. 3 shows an example of the patterned cross-sectional TEM specimen. This pattern 
was deposited with various materials (i.e., metal, oxide and nitride) and the pitch of the Si patterns was very small about $60 \mathrm{~nm}$. Typically, to precisely evaluate the step coverage in the nano-sized hole array, the thickness of the cross-sectional TEM specimen should be much less than $60 \mathrm{~nm}$. Therefore, if we could minimize the curtaining effects during ion milling operation, it is considered that Ar ion milling technique has advantages for preparing specimens as compared to FIB. When the specimen was prepared by a conventional ion milling method (Fig. 3A) the surface of Si was not uniform and thickness modulations were observed, caused by the curtaining effect, as indicated by the arrows. In contrast, in the specimen prepared by backside-ion milling (Fig. 3B), it was seen that the surface was very smooth and flat without any thickness modulations. Therefore, by using the backside-ion milling technique, it is possible to obtain very thin specimens for TEM observation, without damage and curtaining effects. Strictly speaking, the curtaining effect is generated in the conventional ion milling technique as well as in the backside ion milling technique. However, because Ar ions come from the substrate, which is composed of a single component, to the pattern in a predetermined direction, the curtaining effect can be minimized at the interface of the substrate and the pattern.

\section{Backside-lon Milling Combined with FIB Operation for Electron Holography}

Electron holography (Midgley, 2001) is based on TEM; it enables us to visualize the electric field and magnetic flux by measuring the phase information and is a very useful analytical method to quantitatively evaluate the electric and magnetic microstructures in nano-sized materials. However, to obtain the electric and magnetic information by electron holography, the specimen needs to be well controlled with uniform and un-damaged surfaces because the phase information is very sensitive to thickness change and surface damage.

Specimen was $n$-type metal-oxide semiconductor ( $n$-MOS) transistor which comprised the high As doped region about $10^{20} / \mathrm{cm}^{3}$ on the $p$-type Si wafer with the B doping concentration of $10^{15} / \mathrm{cm}^{3}$ and specimen preparation for electron holography was based on the previously mentioned backside-ion milling technique. That is the specimen was fabricated by the backside-ion milling technique, and free space for the reference wave in electron holography was fabricated by FIB operation, as reported by Formanek \& Bugiel (2006) and Yoo et al. (2008).

Fig. $4 \mathrm{~A}$ shows the schematic image of the creation of a hole with dimensions of $12 \times 8 \mu \mathrm{m}$ by FIB. In this step, the Ga ions milled the dummy wafer, glue layer, and some part of the film or patterned side, as indicated the dotted box. Subsequently, to remove the surface damage caused by Ga ions during FIB milling, Ar ion milling was applied for a short period (2 to 3 minutes) at 2 to $3 \mathrm{kV}$. Finally, in order to avoid electrostatic charging of the specimen surface under the electron beam, the specimen surface was coated with a $10 \mathrm{~nm}$ carbon layer. The electron holography study was carried out using a $300 \mathrm{kV}$ FE-TEM installed with a biprism and 2 mega-pixel slow-scan
A

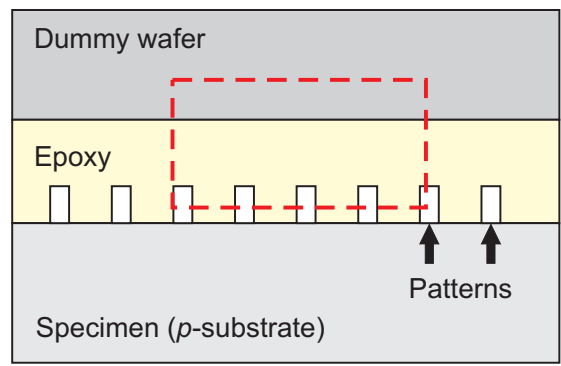

B

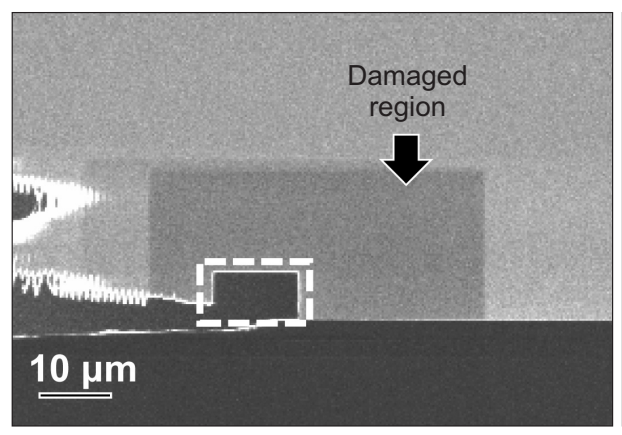

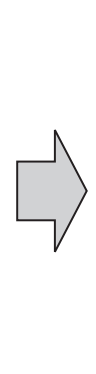

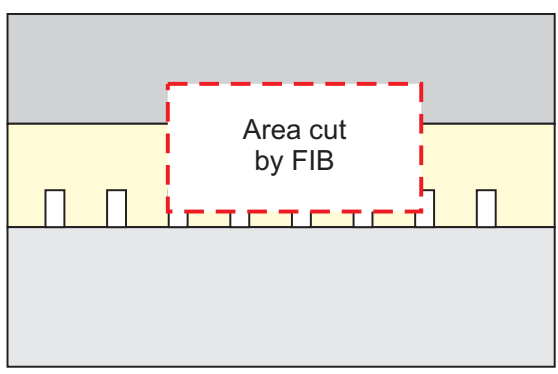

C

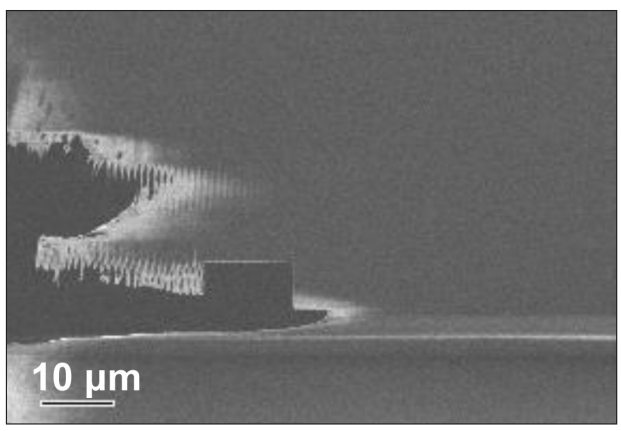

Fig. 4. (A) Schematic illustrations of focused ion beam (FIB) process for creating a free space for electron holography. Scanning electron microscopy images of the specimen surface after creating a hole by FIB (B) and after cleaning process with post-ion milling $(\mathrm{C})$. 

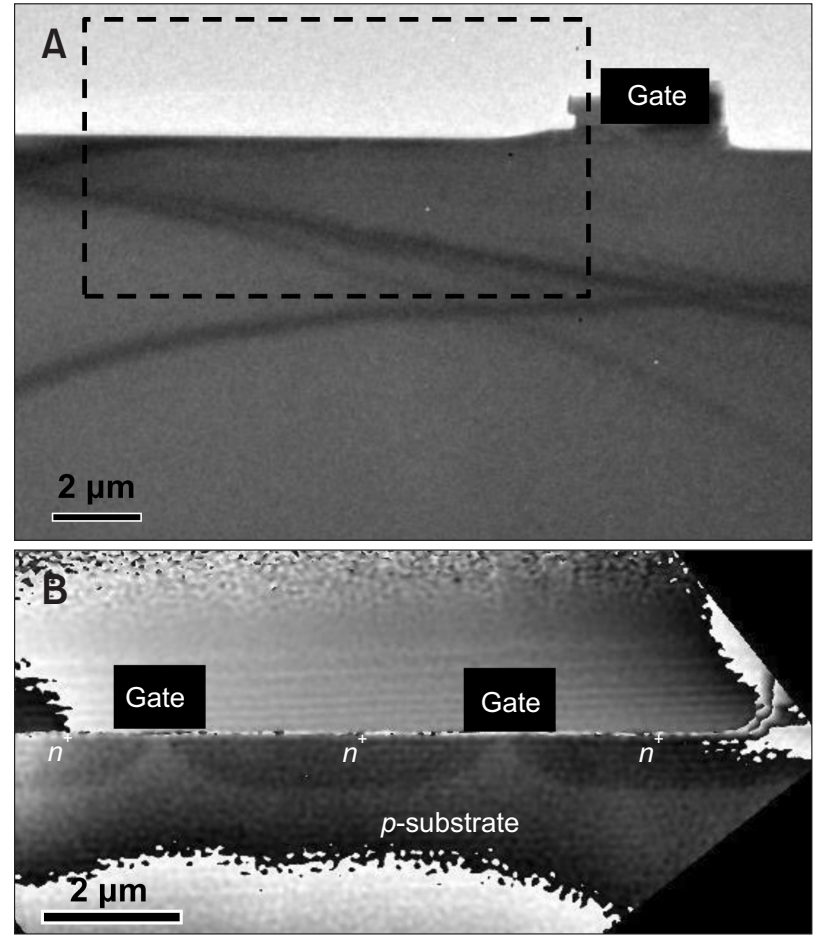

Fig. 5. (A) Transmission electron microscopy bright-field image of the patterned specimen for electron holography. (B) Reconstructed phase image of the dotted box in Fig. 5A prepared by backside-ion milling combined with Focused ion beam operation.

charge-coupled device for recording the electron hologram.

Fig. 4B shows an scanning electron microscope image after creating the hole by FIB for the reference wave. It is shown that the hole and a damaged rectangular region with dark contrast caused by Ga contamination. This damaged region from $\mathrm{Ga}$ contamination could be clearly removed by post $\mathrm{Ar}$ ion milling at 2 to $3 \mathrm{kV}$ (Fig. 4C).

Fig. 5A shows a TEM bright-field image of the n-MOS transistor prepared by back-side ion milling combined with FIB operation. Because the damaged layers are reduced to 5 $\mathrm{nm}$ in thickness after backside-ion milling (Barna et al., 1999), it shows that the obtained specimen has uniform surfaces without curtaining effects and $\mathrm{Ga}$ ion contaminations. A reconstructed phase image which corresponds to the information of phase shifts is shown in Fig. 5B. Here, gates on a Si-wafer was removed by FIB operation and twodimensional (2D) dopant profiles where bright and dark contrasts in Fig. 5B indicate the $p$ - and $n$-doped regions in the specimen, respectively, was clearly demonstrates.

If the specimen was prepared by conventional Ar ion milling technique, it will be difficult to visualize the $2 \mathrm{D} p-n$ junction profiles because of the curtaining effect introducing thickness variations. Moreover, if the specimen was prepared only by the FIB technique, damage caused by the bombardment of $\mathrm{Ga}$ ions will prevent precise analysis.

\section{SUMIMARY}

In this paper, we introduced the backside Ar ion milling technique for the preparation of cross-sectional TEM specimens, and backside-ion milling combined with FIB operation for electron holography. The backside-ion milling technique has advantages of allowing one to prepare crosssectional specimens having thin and uniform surfaces. This technique has very useful applications in the analysis of both the patterned specimen (e.g., 3D integrated circuit, nanoarrayed hole patterns or line pattern with high aspect ratio) or relatively thick layers because of the minimization of the curtaining effect.

By preparing the specimens by back-side ion milling combined with FIB, it is possible to quantitatively analyze the $2 \mathrm{D} p-n$ junction profiles in semiconductor with a large observable area and with the sample quality sufficient for electron holography study.

\section{CONFLICT OF INTEREST}

No potential conflict of interest relevant to this article was reported.

\section{REFERENCES}

Abrahams M S and Buiocchi C J (1974) Cross-sectional specimens for transmission electron microscopy. J. Appl. Phys. 45, 3315.

Barna Á, Pécz B, and Menyhard M (1999) TEM sample preparation by ion milling/ amorphization. Micron 30, 267-276.

Chew N G and Cullis A G (1987) The preparation of transmission electron microscope specimens from compound semiconductors by ion milling. Ultramicroscopy 23, 175-198.

Formanek P and Bugiel E (2006) Specimen preparation for electron holography of semiconductor devices. Ultramicroscopy 106, 365-
375.

Giannuzzi L A and Stevie F A (1999) A review of focused ion beam milling techniques for TEM specimen preparation. Micron 30, 197-204.

John C B and Robert S (1984) The preparation of cross-section specimens for transmission electron microscopy. J. Electron Microsc. Tech. 1, 53-61.

Midgley P A (2001) An introduction to off-axis electron holography. Micron 32, 167-184. 
Mkhoyan K A, Baston P E, Cha J, Schaff W J, and Silcox J (2006) Direct determination of local lattice polarity in crystals. Science 312, 1354, supplementary material.

Okuno H, Takeguchi M, Mitsuishi K, Guo X J, and Furuya K (2008) Sample preparation of GaN-based materials on a sapphire substrate for STEM analysis. J. Electron Microsc. 57, 1-5.

Orloff J, Utlaut M, and Swanson L (2003) High Resolution Focused Ion Beams: FIB and Its Applications (Kluwer Academic/Plenum Publishers, New York).

Preble E A, McLean H A, Kiesel S M, Miraglia P, Albrecht M, and Davis P F (2002) Application of Nomarski interference contrast microscopy as a thickness monitor in the preparation of transparent, SiC-based, cross-sectional TEM samples. Ultramicroscopy 92, 256-271.

Stevie F A, Shane T C, Kahora P M, Hull R, Bahnck D, Kannan V C, and David E (1995) Applications of focused ion beams in microelectronics production, design and development. Surf. Interface Anal. 23, 61-68.

Traeholt C, Wen J G, Svetchnikov V, Delsing V, and Zandbergen H W (1993) A reliable method of TEM cross section specimen preparation of YBCO films on various substrates. Physica C 306, 318-328.

Yoo J H, Yang J M, Shaislamov U, Ahn C W, Hwang W J, Park J K, Park C M, Hong S B, Kim J J, and Shindo D (2008) Electron holography study for two-dimensional dopant profile measurement with specimens prepared by backside-ion milling. J. Electron Microsc. 57, 13-18. 